Recepción: 20 / 04 / 2017

Aceptación: 20 / 05 / 2017

Publicación: 15 / 09 / 2017

Ciencias de la computación

\title{
Aplicación Web para la gestión comercial de la empresa VisanvitalCia. Ltda
}

Web application for the commercial management of the. company VisanvitalCia. Ltda.

\section{Aplicativo da Web para o gerenciamento comercial do VisanvitalCia. Ltda}

\author{
Antonio R. Sanchez-Nuñez ${ }^{\text {I }}$ \\ asanchezn@ups.edu.ec \\ Byron V. Guerrero-Rodriguez II \\ bvguerrero@uce.edu.ec \\ Gerardo M. Llerena-Carrera ${ }^{\text {III }}$ \\ gmllerenac@uce.edu.ec
}

Correspondencia: gmllerenac@uce.edu.ec

I. Diploma Superior en Sistemas de Información Empresarial; Especialista en Redes de Comunicación de Datos; Magister en Informática Empresarial; Master of Science in Leadership; Ingeniero en Electrónica y Telecomunicaciones; Universidad Politécnica Salesiana, Cuenca, Ecuador.

Diploma Superior en Docencia Universitaria; Master of Science in Leadership; Ingeniero Electrónico en Control y Automatización; Universidad Central del Ecuador, Quito, Ecuador.

III. Magister en Administración de Empresas (MBA) Programa Integral de Habilidades Multiples; Ingeniero en Geologia; Universidad Central del Ecuador, Quito, Ecuador. 


\section{Resumen}

En el presente trabajo investigativo es un resumen de una solución tecnológica dada al problema relacionado con la disminución en las ventas de la empresa VisanVitalCia. Ltda.Se recopiló información sobre la problemática, se revisó los antecedentes investigativos y también se planteó los objetivos así como la justificación respectiva.Se recoge los aspectos principales relacionados con el fundamento científico en el que se basa la solución planteada, esos aspectos científicos tienen que ver con sistemas de información, páginas web, bases de datos y más.

Se sintetiza la investigación de campo realizada y se plasman los resultados obtenidos, allí se ratifican los problemas descritos en la introducción y se reitera que la empresa tiene dificultades en las ventas debido a su reducido segmento de mercado, así como a los costos elevados de promoción.Se plantea la plataforma web como elemento canalizador para ampliar el segmento de mercado así como también para hacer promoción a muy bajo costo. Las herramientas utilizadas son las denominadas de software libre y la propuesta se halla funcionando en el Internet.

Palabras claves: Aplicación web; gestión; promoción; ventas. 


\section{Abstract}

The following research intends to provide technological solution to a problem associated with sales decrease.Information about the problem has been collected and show the main aspects related to scientific foundation where the solution will be based; those scientific aspects deal with information systems, websites, databases like and some others.

The field research carried out and the results obtained are synthetized, confirming the problems described in the introduction and reiterating that the company has difficulties in sales due to its small market segment, as well as the high costs of promotion. The platform web is posed as a channel element in order to extend the market segment as well as to advertise with lower costs. The tools used are called free software and the proposal is currently running on the internet.

Key words: Web Application; management; promotion. 


\section{Introducción.}

Se realizó inicialmente una investigación en la biblioteca de la Universidad Regional Autónoma de Los Andes, así como en algunos repositorios digitales de varias instituciones de educación superior en el país, encontrándose con algunos trabajos de grado a nivel de maestría que sirven como antecedente previo, entre ellos podemos mencionar los siguientes (Kendall \& Kendall, 2011) (Oros, 2007):

El trabajo de los Ingenieros Torres Vargas Richard y Saltos Viteri Harry con el tema: “Sistema Informático basado en tecnología WAP para atención al usuario de la Empresa Eléctrica Los Ríos” presentado en el año 2009 con lo que se determina que las aplicaciones web para móviles son parte de las nuevas tendencias tecnológicas de acceso a los contenidos con los que interactúan los usuarios. (Angulo, 2010)

También en el repositorio de la Escuela Politécnica de Chimborazo se consultó el trabajo de titulación de (Carrazco Sanunga, 2012) en el cual se diagnostica las deficiencias que tiene la empresa hotelera en la atención a sus clientes.

A nivel internacional se analizó el trabajo de (Claros Rodríguez \& Willatt, 2013) y en el cual se analiza los nuevos mercados a los cuales puede acceder la empresa y la estructura tecnológica para ello. Esta empresa vende productos innovadores y ha tomado como mercado a todo Chile, también se describe la gestión de proceso en cuanto a cobros y entrega a domicilio. (Firtman \& Maximiliano, 2010) 
En resumen se puede señalar que la automatización de los procesos de comercialización vía web son importantes y contribuyen a una ampliación del segmento de mercado en el que trabaja la empresa y con eso posibilita el aumento de las ventas de la misma.

La empresa "Visan Vital" es un emprendimiento nacido de la iniciativa de su gerente propietario, en base a su conocimiento sobre insumos médicos debido a sus trabajos anteriores como visitador médico y por sus estudios iniciales en esta área. De las visitas realizadas al micro empresa y también en base a las conversaciones mantenidas con su gerente, se han podido detectar las siguientes dificultades en la gestión comercial de la misma, así tenemos:

- Los niveles de comercialización de insumos médicos, ha ido disminuyendo debido a la elevada competencia existente y especialmente por los grandes incrementos en los costos de las mismas

- La empresa no ha podido ampliar sus segmentos de mercado debido a que debe pagar elevadas comisiones y gastos en transporte, lo que ha producido que el mercado de la misma se haya reducido a la ciudad de Quito.

- También la empresa gasta elevadas sumas de dinero en promoción radial, así como en afiches, los cuales tienen efecto momentáneo y de muy corto alcance

- La empresa oferta como complemento a sus ventas los procesos de asistencia técnica y capacitación, para ello coordina el desplazamiento de personal experto en el área, lo cual también implica gastos elevados, sobre todo para procesos de capacitación

Todo lo señalado anteriormente lleva a afirmar que se tiene problemas de comercialización dentro de la gestión comercial de la empresa. 
Para dar solución a la problemática se planteó el objetivo de presente estudio que es desarrollar e implementar una aplicación web que tiene como objetivo permitir el mejoramiento de la gestión comercial de la empresa "Visan Vital” de la ciudad de Quito.

\section{Materiales y métodos.}

La modalidad investigativa que se ha utilizado en este proyecto es la denominada cualicuantitativa. La investigación cualitativa es el procedimiento metodológico que se caracteriza por entender el conjunto de cualidades interrelacionadas que caracterizan a un determinado fenómeno, se la aplico para determinar las características del problema como la imposibilidad de que los clientes hagan sus compras rápidamente y con ello elevar las mismas para la empresa.

La investigación cuantitativa se caracteriza por recoger, procesar $\mathrm{y}$ analizar datos cuantitativos o numéricos sobre variables previamente determinadas. Dicha metodología se la aplico para determinar estadísticamente los síntomas de la problemática relacionada con las ventas en la empresa.

Los tipos de investigación aplicados son:

Bibliográfica: Este tipo de investigación se la desarrolla en base a la recopilación de la información de fuentes diversas, se la utilizó para desarrollar el marco teórico orientado esencialmente a herramientas de software libre como Mysql, apache y php.

De Campo: se la lleva a cabo en base a encuestas o entrevistas y se la aplico para desarrollar el marco metodológico. Se entrevistó al Sr. Sánchez gerente propietario de la empresa Visanvital y se encuesto tanto a los clientes como a los empleados. 
Se define como la muestra, a un porcentaje de la población y la fórmula de cálculo es dada por [1]:

$$
\text { Muestra }=\frac{\text { Población }}{\left(\text { Población-1)error }{ }^{2}+1\right.} .
$$

La población involucrada en la problemática de este trabajo investigativo está estructurada de la siguiente forma:

Tabla1

Población Investigada

\begin{tabular}{|l|l|}
\hline Función & Número \\
\hline Gerente de la empresa & 1 \\
\hline Empleados & 10 \\
\hline $\begin{array}{l}\text { Clientes frecuentes promedio } \\
\text { mensual }\end{array}$ & 80 \\
\hline TOTAL & 91 \\
\hline
\end{tabular}

En este caso como la población es baja (inferior a 100) se asume toda ella como la muestra.

Las técnicas de investigación aplicadas fueron: entrevista al señor gerente y encuestas tanto a empleados como a clientes.

Los instrumentos utilizados fueron: Guía de entrevista para la gerente y cuestionarios específicos para clientes y empleados.

Luego de realizada la investigación de campo se procedió a tabular los resultados de las encuestas, los cuales se detallan se obtuvieron las siguientes conclusiones: 
- Los clientes destinan entre 2 y 3 horas para realizar la compra, este tiempo entre el contacto y la visita del vendedor

- La mayoría de clientes gasta entre 3000 y 5000 dólares mensuales en sus compras

- $\quad$ Aunque el número de clientes se ha mantenido, hay una tendencia a la baja y a la reducción de ventas

- La promoción de productos es realizada de forma tradicional, con alcance limitado y con gastos elevados

- No se utilizan nuevos medios de promoción y comercialización como es el internet.

- Se debe buscar medios alternativos de comercialización para ampliar mercados, incrementar clientes y aumentar ventas.

La investigación de campo realizada, ratifica la problemática relacionada con el descenso de las ventas y con el elevado gasto en publicidad, esto significa que la solución tiene que orientarse a esos dos aspectos, por lo tanto se propone como solución a la problemática la implementación de un portal web que sirva esencialmente para realizar comercio electrónico y para promocionar la empresa VisanVital. La propuesta se esquematiza en la figura [2]. 


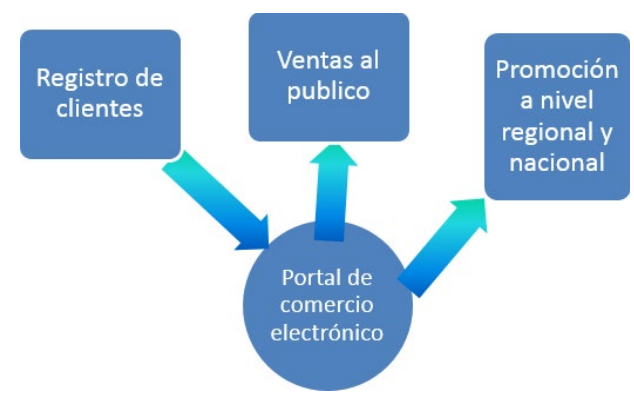

Figura. 2. Propuesta para VisanvitalCia. Ltda.

Del trabajo de campo también se deduce que la empresa requiere una aplicación web que le permita vender los productos, mantener el catalogo actualizado y que los clientes puedan empaparse de las novedades. Esta aplicación debe contribuir a la gestión comercial de la empresa en los ámbitos que muestra la figura [3].

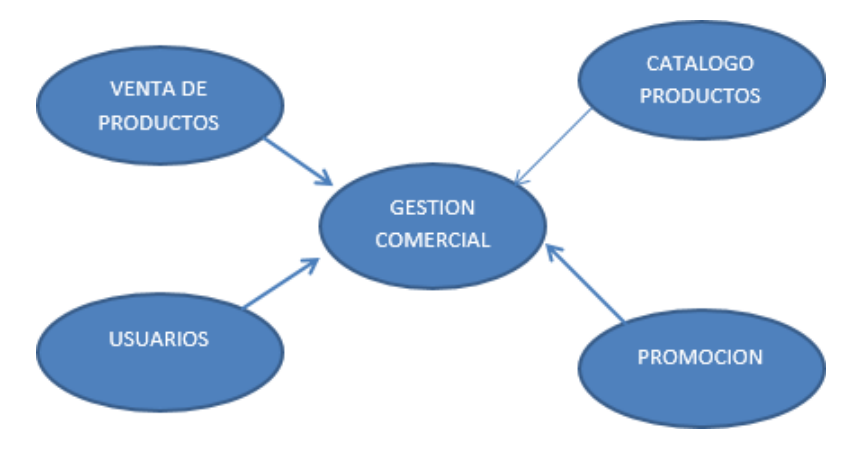

Figura. 3. Ámbitos de la gestión comercial de VisanvitalCia. Ltda.

La metodología de desarrollo de software seleccionada para elaborar el portal web es la denominada lineal o en cascada. Esta metodología define una organización secuencial de las etapas del desarrollo de software, se debe hacer inicialmente un listado de requerimientos que surgen del análisis inicial o previo, luego se diseña los aspectos relacionados a la base de datos y a las interfaces del sistema. Seguidamente se procede a desarrollar la codificación respectiva de cada interface diseñada, luego de esto se hacen las pruebas respectivas y finalmente se implementa el 
software.

Hay que señalar que en cada fase debe realimentarse para que se mejoren los procesos y por ende la automatización sea de mejor manera. En cuanto a las herramientas de desarrollo, se ha considerado que las más viables para el desarrollo del portal web son: Wordpress, Php, Apache, MySq1.

La representación de los procesos operativos que contempla el sistema son: gestión del usuario de internet representado en la figura [4], gestión del usuario vendedor representado en la figura [5], gestión del usuario administrador representado en la figura [6] y diagrama de secuencia de los usuarios representado en la figura [7].

El sistemaconsta de los siguientes Módulos:

- $\quad$ Tienda. Permite al usuario puede mirar los productos existentes

- Categorías.Permite que el usuario pueda realizar altas, bajas, modificaciones consultas sobre las categorías de los productos.

- Mi cuenta.Permite acceder a la información de clientes, se puede identificar y también registrar

- Carrito.Contiene un listado de productos seleccionados por el cliente.

- La empresa. El sistema muestra la información empresarial. 
Antonio R. Sanchez-Nuñez; Byron V. Guerrero-Rodriguez; Gerardo M. Llerena-Carrera

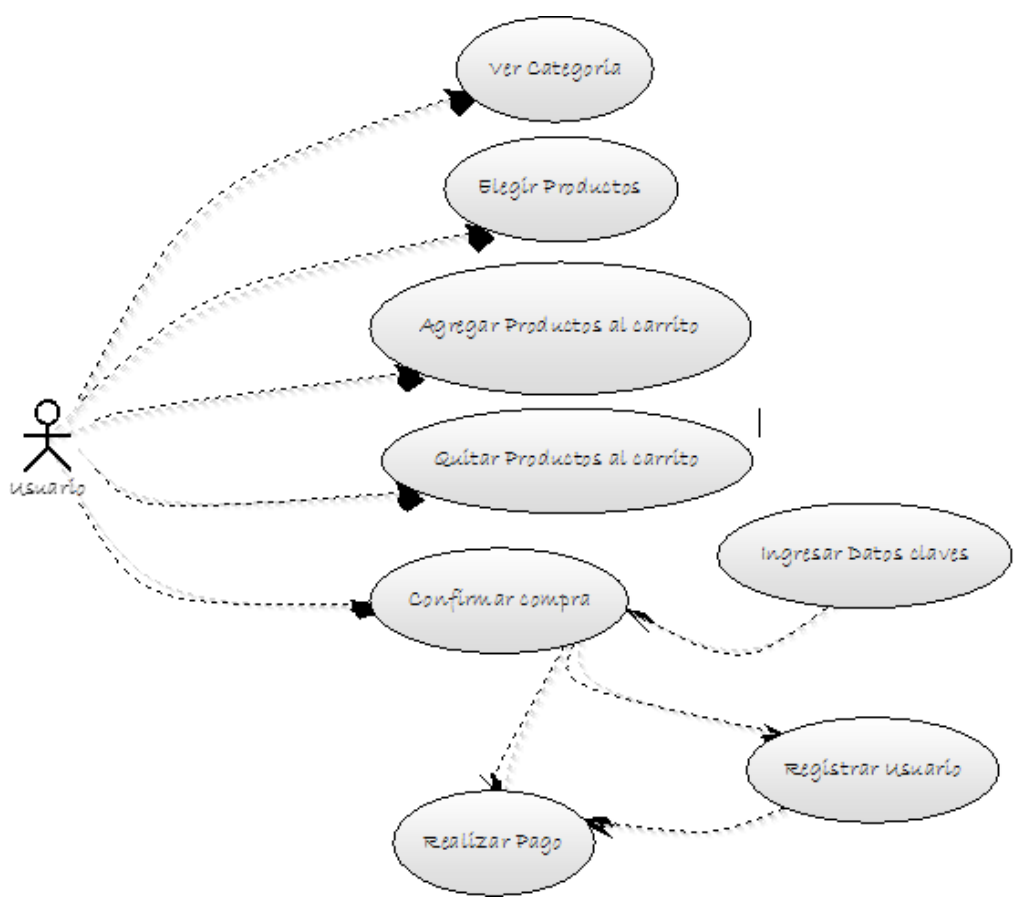

Figura. 4. Casos de uso de la gestión del usuario de internet.

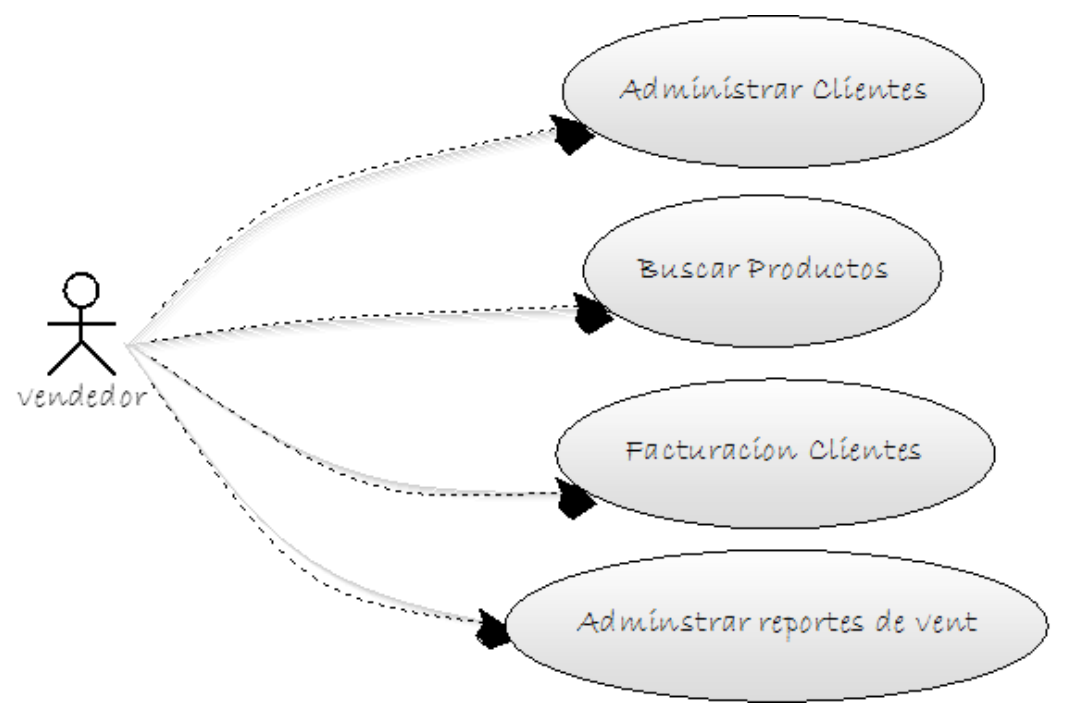

Figura. 5. Casos de uso de la gestión del usuario vendedor. 
Aplicación Web para la gestión comercial de la empresa VisanvitalCia. Ltda

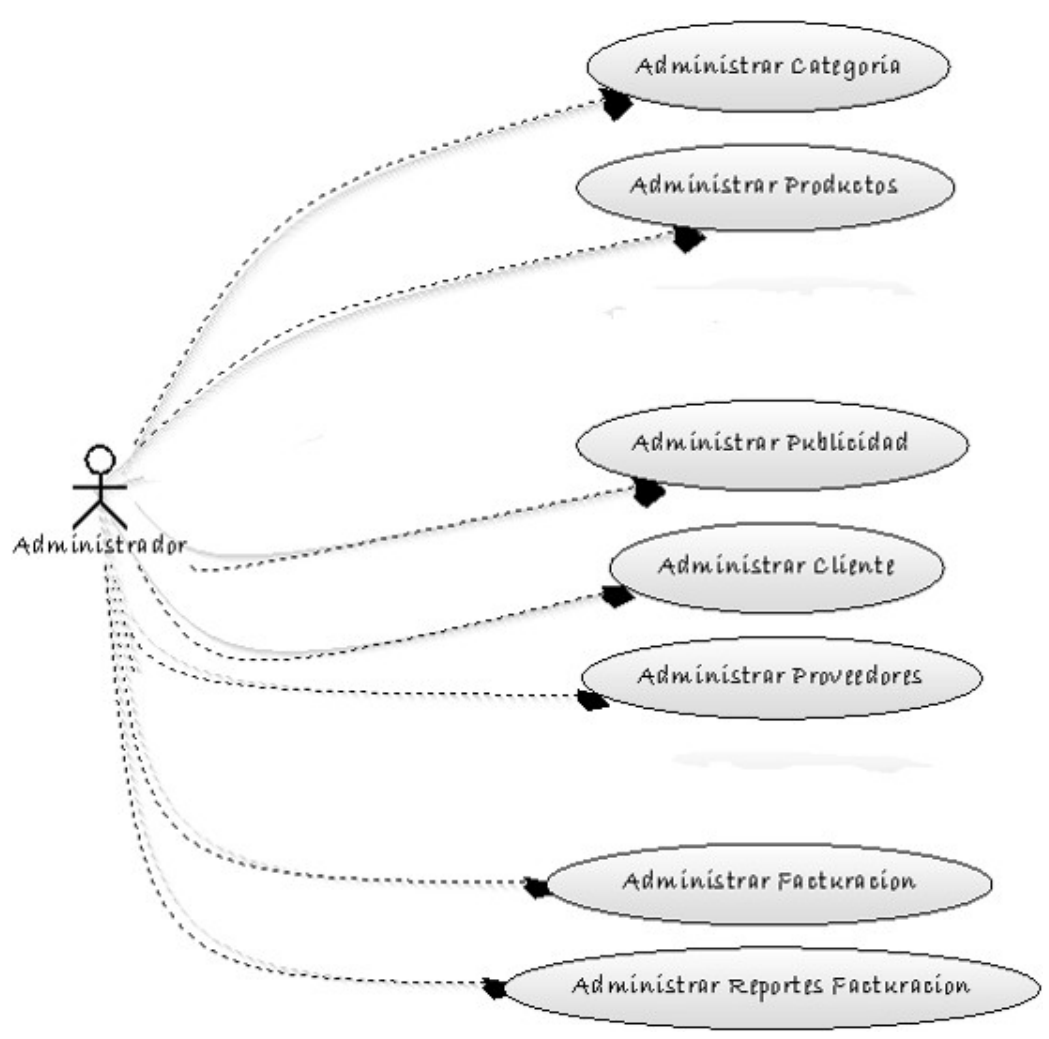

Figura. 6. Casos de uso del usuario administrador.

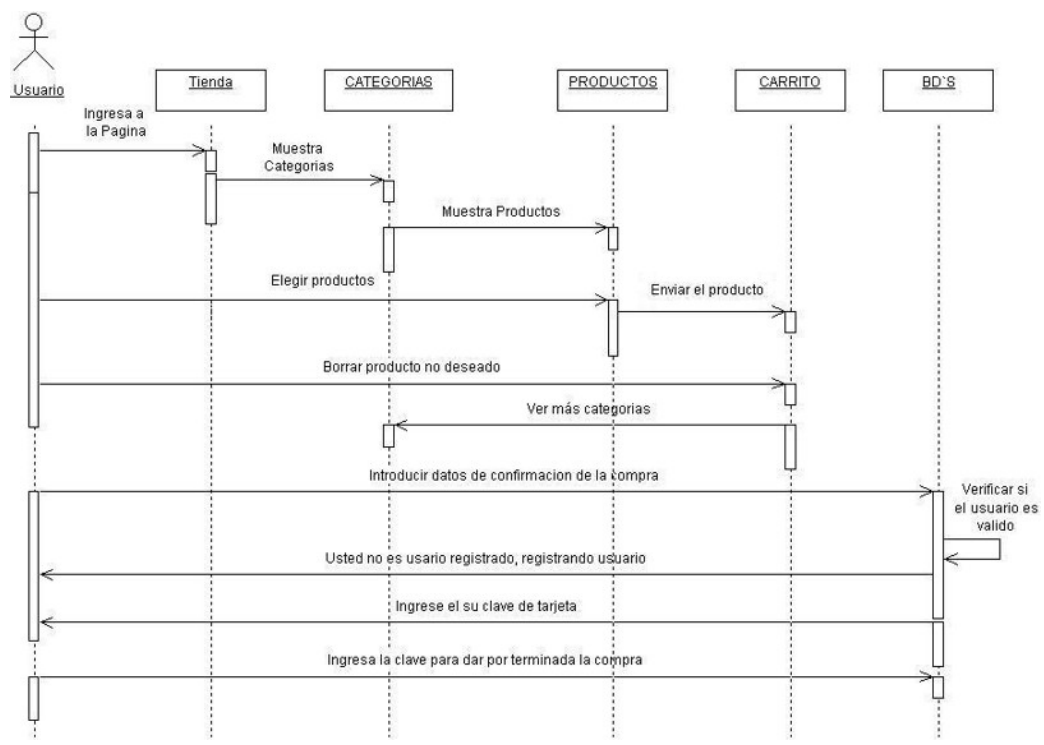




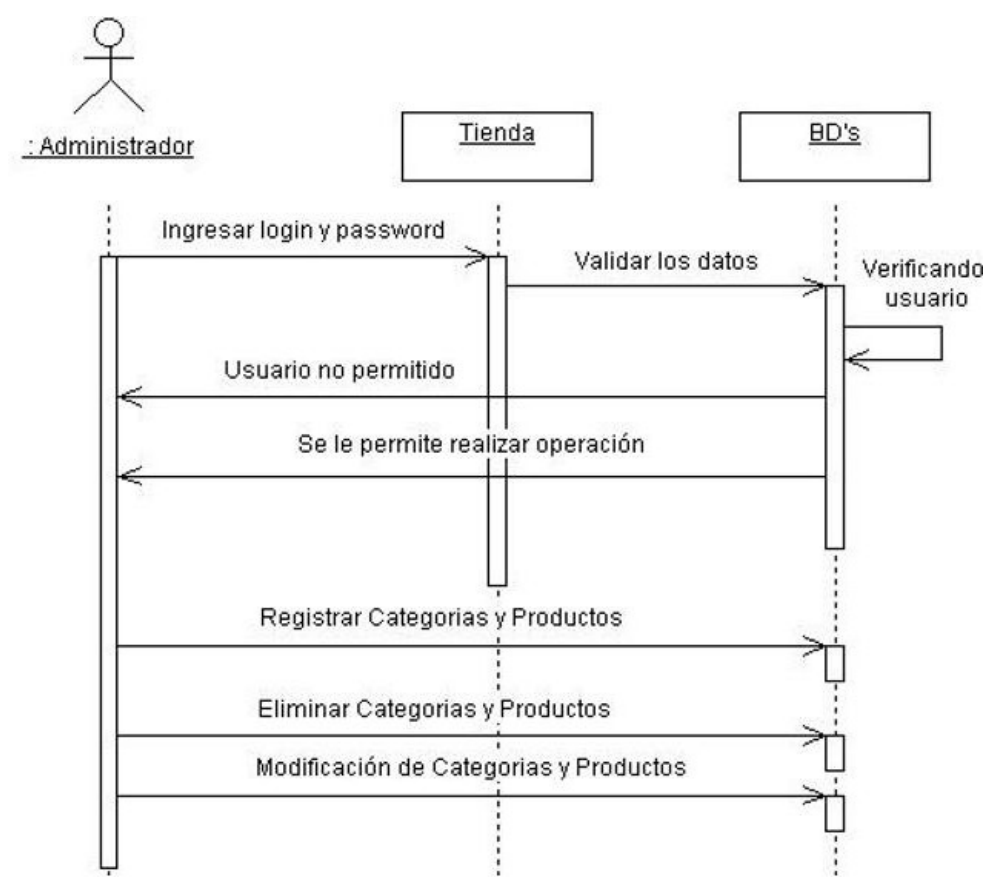

Figura. 7. Secuencia de los Usuarios.

El portal web funciona en el dominio http://visanvital.com/, para ello se ha conseguido un hosting con tecnología Linux, el mismo que soporta las herramientas con las que se desarrolló el software. Dichas herramientas son PHP, Apache, MySql y JavaScript y wordpress.

Para la subida de archivos se utiliza una conexión FTP provista por el administrador del hosting.

En cuanto a los requerimientos de hardware para navegar se tiene: Pc con Microprocesador P4 de 3.0 Mhz o superior, Teclado estándar, Mouse estándar, Monitor, Disco duro (espacio de 500Gb), Memoria Ram 2Gb. 


\section{Resultados.}

Las pruebas permanentes durante el desarrollo del sistema realmente constituyen un factor importante de mejora continua, el software desarrollado ha sido sometido a las siguientes pruebas:

Caja Negra: Esta prueba esencialmente permitió verificar la idoneidad de los resultados, se aplicó para validar los saldos del kardex luego de los ingresos y egresos realizados, se ingresó los datos de los clientes, se hicieron las ventas y se verifico los saldos respectivos. Aquí, no se analizó los procesos, solo se verifico la validez de las Frecuencias, la figura [8] y [9] muestran una prueba al inventario antes y después de las ventas respectivamente.

Se realizaron 3 ventas de un producto como prueba con un total de 10 productos vendidos, y se observa que el sistema el resultado cuadra con las cantidades disponibles antes y después de las ventas.

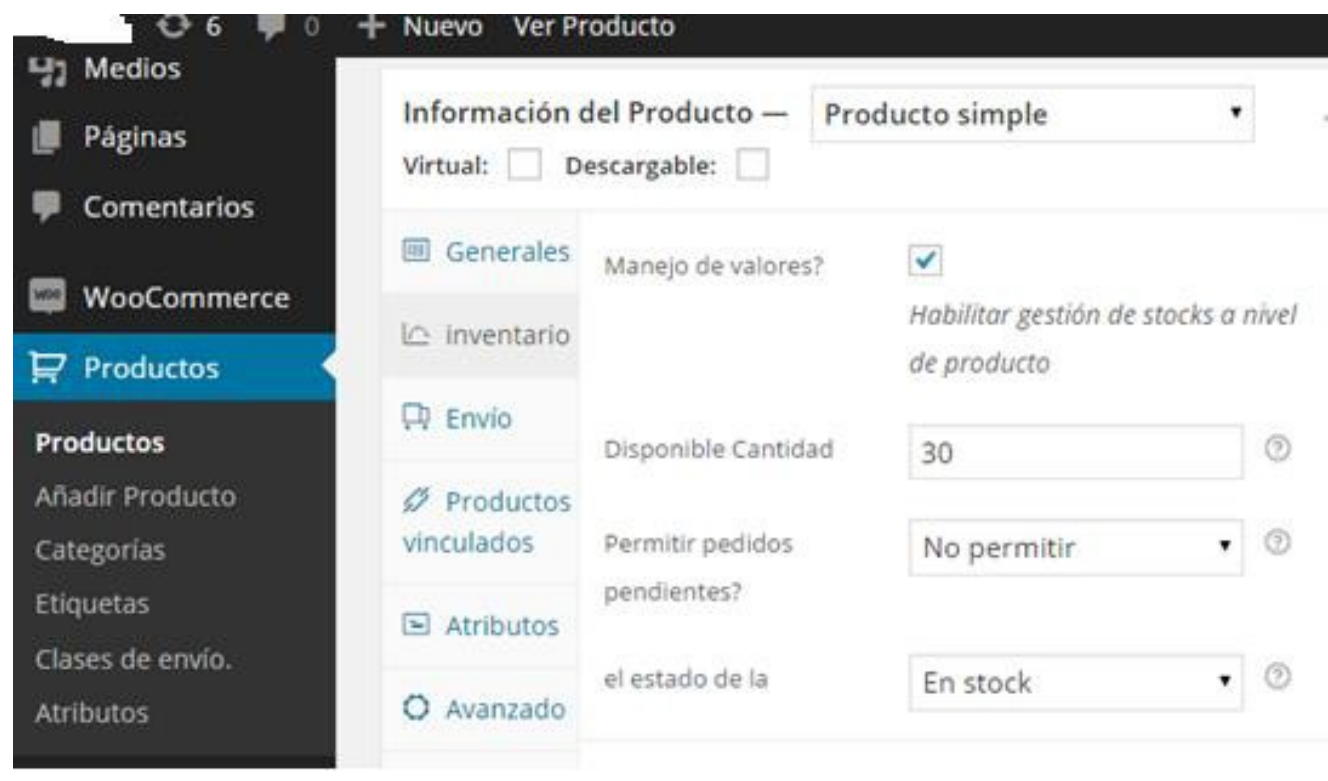

Figura. 8. Prueba de caja negra al inventario antes de las ventas. 
Antonio R. Sanchez-Nuñez; Byron V. Guerrero-Rodriguez; Gerardo M. Llerena-Carrera

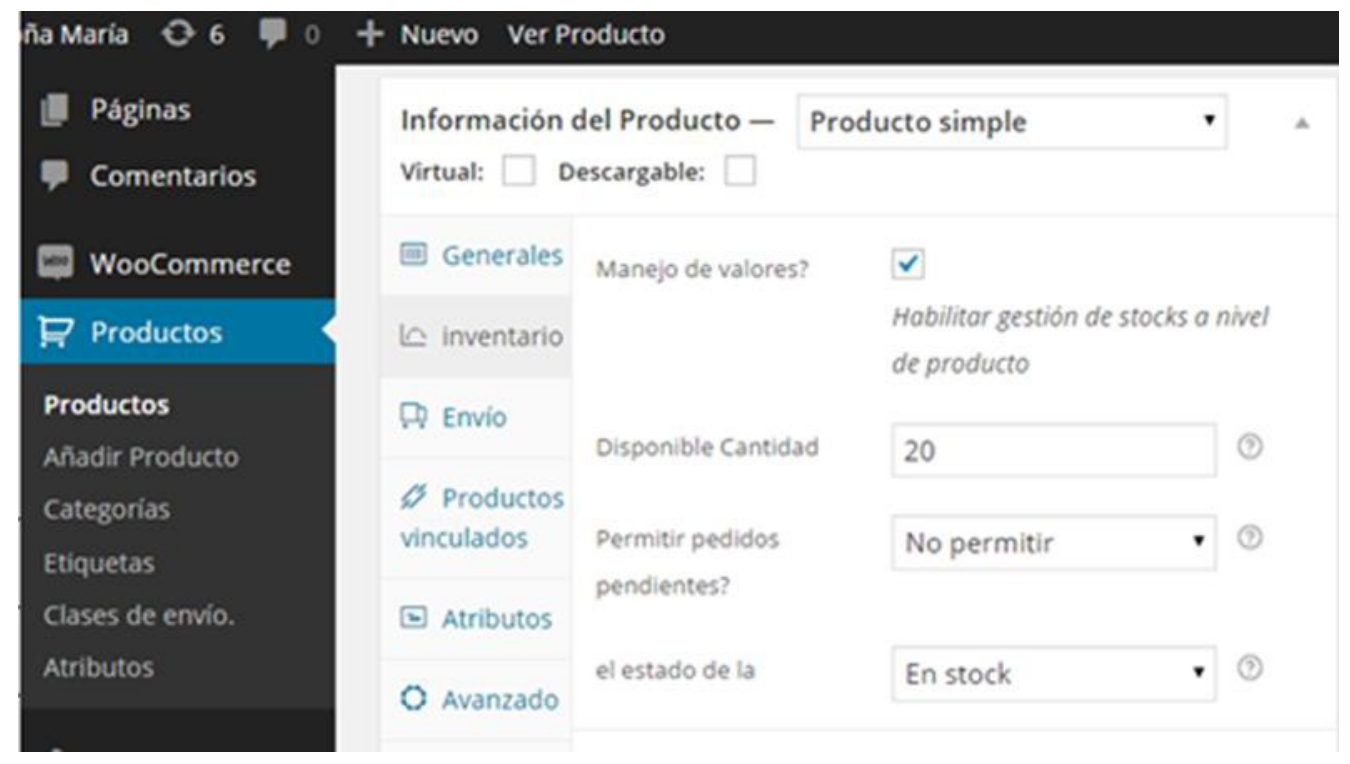

Figura. 9. Prueba de caja negra al inventario después de las ventas.

Caja blanca: Para esta prueba se utilizó la herramienta de depuración que incorpora Google Crome en la pestaña de herramientas para desarrolladores. La figura [10] muestra la captura de la herramienta de depuración, y en la tabla [2] se puede observar el resultado.

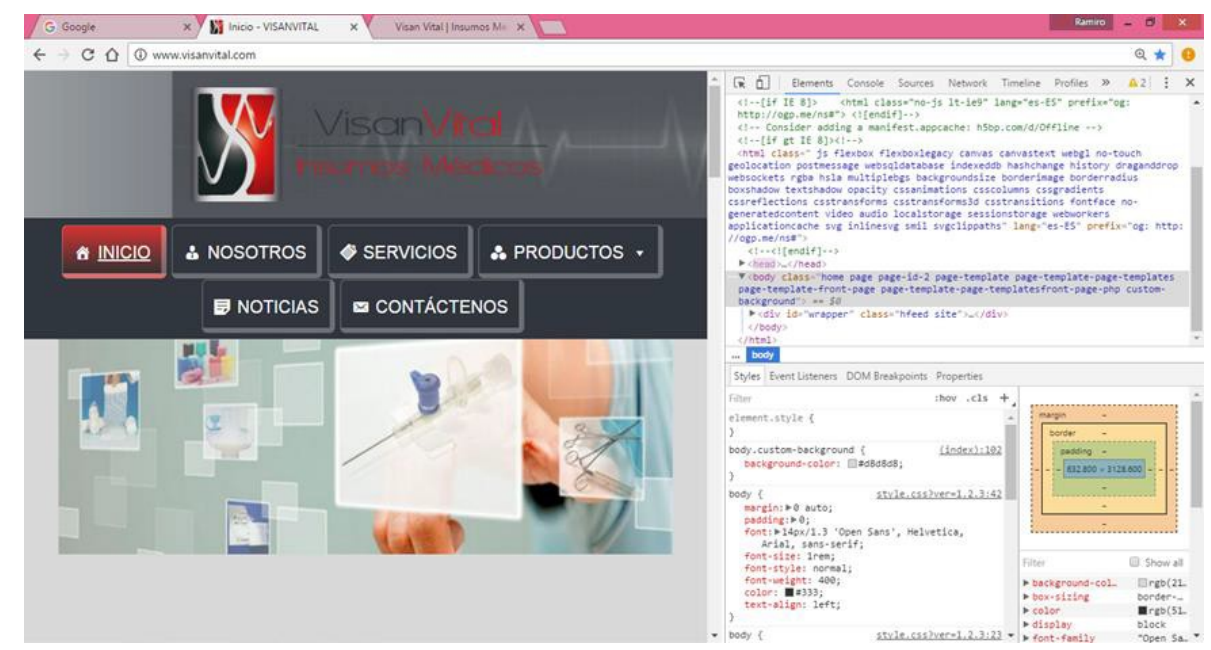

Figura. 10. 
Tabla 2

\section{Resultados de caja negra}

\begin{tabular}{|l|c|l|c|}
\hline \multicolumn{1}{|c|}{ Página } & Tipo & Descripción & Observación \\
\hline $\begin{array}{l}\text { http://visanvit } \\
\text { al.com/index }\end{array}$ & Inicial & $\begin{array}{l}\text { Muestra } \\
\text { acceso al } \\
\text { portal }\end{array}$ & Funciona \\
\hline$\underline{\text { http://visanvit }}$ & $\begin{array}{c}\text { Intern } \\
\text { al.com/micue } \\
\text { nta }\end{array}$ & $\begin{array}{l}\text { Muestra los } \\
\text { productos } \\
\text { elegidos }\end{array}$ & Funciona \\
\hline
\end{tabular}

Con las dos pruebas se puede verificar el correcto funcionamiento de la aplicación implementada para las dos secciones seleccionadas para este ejemplo. El mismo procedimiento se lo realizó para cada sección que forma parte de la aplicación verificando su correcto funcionamiento.

\section{Conclusiones.}

La comercialización electrónica y el marketing digital permiten la ampliación de los segmentos de mercado y por ende la posibilidad de aumentar el potencial número de clientes, la empresa que lo usa tiene enormes posibilidades de aumentar sus ventas.

Cuando se realizan aplicaciones que funcionen bajo el entorno web, se debe pensar en algunos tipos de seguridades, especialmente relacionadas con la inyección de código malicioso. También se debe tomar muy en cuenta la verificación del pedido, así como del pago antes de realizar la entrega del producto.

El portal web permitirá obtener algunos beneficios para la empresa, entre ellos podemos señalar: Ampliación de mercados posibles, esto debido a que el internet llega a todos los sitios y 
cada usuario de la red puede considerarse como un potencial cliente. Promoción permanente, esto debido a que se tiene 24 horas y 365 días al año de información promocional a muy bajo costo

\section{Bibliografia.}

Angulo, L. (2010). Disenos de Paginas Web. Lima: Macpro.

Carrazco Sanunga, N. (2012). Evaluación de la calidad del servicio y atención al cliente en la Hostería Karen de la ciudad de Guano. Riobamba: Escuela Superior Politécnica de Chimborazo.

Claros Rodríguez, N., \& Willatt, C. (2013). Plan de negocios para una empresa de E-commerce comercializadora de productos novedosos. Santiago: Universidad de Chile.

Firtman, L., \& Maximiliano, A. (2010). AJAX - Web 2.0 Con jQuery Para Profesionales (Spanish Edition). México: Alfaomega.

Kendall, K., \& Kendall, J. (2011). Análisis y diseño de sistemas. México: Person.

Oros, J. C. (2007). Diseño de Paginas Web con XHTML, JAVASCRIPT y CSS - Navegar en Internet (Spanish Edition). México: Alfaomega Grupo Editor. 